\title{
COHOMOLOGIES OF HOMOGENEOUS ENDOMORPHISM BUNDLES OVER LOW DIMENSIONAL KÄHLER $C$-SPACES
}

\author{
By \\ Masaro TAKAHASHI
}

\section{Introduction}

In this paper, we determine the infinitesimal deformations of an EinsteinHermitian structure of a homogeneous vector bundle in several cases. In particular, we get the tangent space at the homogeneous structure of the moduli space of Einstein-Hermitian structures as the representation space of a compact Lie group.

A compact simply connected homogeneous Kähler manifold is called a Kähler $C$-space. Such a manifold can be written as $G / K$ where $G$ is a compact semisimple Lie group and $K$ is the centralizer of a toral subgroup of $G([10])$. Let $G^{C}$ be the complexification of $G$ and $K^{C}$ the complexification of $K$ in $G^{C}$. We denote by $L$ the parabolic subgroup of $G^{c}$ which contains $K^{c} . G / K$ is diffeomorphic to $G^{c} / L$. Thus $G / K$ admits a holomorphic structure from the holomorphic structure of $G^{C} / L$. Moreover it admits a $G$-invariant Kähler metric.

Let $(\rho, V)$ be a complex representation of $K$. Then $(\rho, V)$ can be extended to a holomorphic representation $\left(\rho_{L}, V\right)$ of $L$. The homogeneous vector bundle $E_{\rho}=G \times{ }_{\rho} V$ is isomorphic to the homogeneous holomorphic vector bundle $E_{\rho_{L}}=G^{c} \times{ }_{\rho_{L}} V$ as $C^{\infty}$-vector bundles. Thus the homogeneous vector bundle $E_{\rho}$ has a natural holomorphic structure from the holomorphic structure of $E_{\rho_{L}}$ $([3])$. Moreover if $(\rho, V)$ is irreducible, then $E_{\rho}$ has a unique $G$-invariant Einstein-Hermitian structure up to a homothety ([8]).

An irreducible complex representation $(\rho, V)$ is determined by the highest weight. Then a homogeneous vector bundle $E_{\rho}$ is determined by the highest weight of $(\rho, V)$, if $(\rho, V)$ is irreducible. It is natural to ask how we describe the deformations of the holomorphic structure and the Einstein-Hermitian structure by the highest weight. Also we ask how we describe moduli spaces of holomorphic structures and Einstein-Hermitian structures by the highest weight.

Received January 17, 1994. 
In the deformation theory of complex structures of complex manifolds, the complex structure of a Kähler $C$-space is locally stable ([3]). But the holomorphic structure of a homogeneous vector bundle generally is not locally stable. Here the local stability means that any deformation space is trivial. So there is a problem to find sufficient conditions for the local stability of the holomorphic structure of a homogeneous vector bundle.

Let $\operatorname{End}\left(E_{\rho}\right)$ be the endomorphism bundle of $E_{\rho}$ and $\mathfrak{g l}\left(E_{\rho}\right)$ the subbundle of $\operatorname{End}\left(E_{\rho}\right)$ which consists of trace free endomorphisms. It is well known that the Dolbeault cohomology group $H^{0.1}\left(G / K, \operatorname{End}\left(E_{\rho}\right)\right)$ is the tangent space of the moduli space of holomorphic structures if $H^{0.0}\left(G / K, \mathfrak{g l}\left(E_{\rho}\right)\right)=H^{0.2}\left(G / K, \operatorname{al}\left(E_{\rho}\right)\right)$ $=\{0\}$. The moduli space of Einstein-Hermitian structures is an open subset of the moduli space of holomorphic structures. Under the same conditions, $H^{0,1}\left(G / K, \operatorname{End}\left(E_{\rho}\right)\right)$ is also the tangent space of the moduli space of EinsteinHermitian structures ([7], [6] and [9]). So we think that it is important to compute these cohomologies for our problems.

In this paper, for a first step of problems above we investigate Kähler $C$-spaces $G / K$ with rank $G=2$. In $\S 2$ we shall review a construction of Kähler $C$-spaces and some properties of vector bundles over them. We shall state our results in $\S 3$ and prove them in $\S 4$. Our main results are Theorems 4,5 and Corollary 6 . In the case of $\operatorname{rank} G=2$, we compute $H^{0, p}\left(G / K\right.$, $\left.\operatorname{End}\left(E_{\rho}\right)\right)$ and $H^{0, p}\left(G / K, \operatorname{gl}\left(E_{\rho}\right)\right)$ from the highest weight of $(\rho, V)$ (Theorems 4 and 5$)$. Then we get dimension of the moduli space of Einstein-Hermitian structures of a homogeneous vector bundle in several cases (Corollary 6). The following theorem is an immediate consequence of these results.

Theorem 1. Let $G / K$ be a Kähler $C$-space where $G$ is of type $A_{2}$ or $B_{2}$. Let $(E, h)$ be an irreducible Einstein-Hermitian homogeneous vector bundle over $G / K$ with rank $E=r$. Then the dimension of the moduli space of irreducible Einstein-Hermitian structures of $E$ is as follows:

(1) If $G$ is of type $A_{2}$ or $B_{2}$ and if $K$ is a maximal torus, then the dimension of the moduli space is 0 .

(2) If $G / K \cong S U(3) / S(U(1) \times U(2)) \cong P_{2} C$, then the dimension of the moduli spoce is

$$
\frac{1}{2} \sum_{k=1}^{r-1}(2 k+1)(k+2)(k-1) .
$$

(3) If $G / K \cong S p(2) /(U(1) \times S p(1)) \cong P_{3} C$, then the dimension of the moduli space is

$$
\frac{1}{3} \sum_{k=1}^{r-1}(2 k+3)(2 k+1)(2 k-1)
$$


(4) If $G / K \cong S O(5) /(U(1) \times S O(3)) \cong Q_{3}(C)$, then the dimension of the moduli space is

$$
\frac{1}{2} \sum_{k=1}^{r-1}(2 k-1)(k+2)(k-1)
$$

The author would like to express his gratitude to Professor Mitsuhiro Itoh and Professor Hiroyuki Tasaki for their valuable advices and encouragement.

\section{Preliminaries}

In this section, we review a construction of Kähler $C$-spaces and some properties of homogeneous bundles over them. We refer the reader to the books [1] and [5] for the representation theory of compact Lie group.

Following Wang ([10]), we call a compact simply connected homogeneous Kähler manifold a Kähler C-space. A vector bundle $E$ over a homogeneous space $G / K$ is said to be homogeneous if it is associated to the principal $K$-bundle $G \rightarrow G / K$.

Let $G$ be a compact simply connected semisimple Lie group. Let $T_{0}$ be a toral subgroup of $G$ and $K$ the centralizer of $T_{0}$ in $G$. Then $G / K$ is a compact simply connected homogeneous manifold. Let $T$ be a maximal torus of $G$ which contains $T_{0}$. Then $T$ is contained in $K$ and we put $l=\operatorname{dim} T$. We denote by $\Delta$ the set of nonzero roots of $G$ relative to $T$. Let $\Pi=\left\{\alpha_{1}, \alpha_{2}, \cdots, \alpha_{l}\right\}$ be a fundamental system of $\Delta$. We may assume that $\Pi$ is the system of simple roots of $\Delta$ for a suitable order of the Lie algebra of $T$. In this order, we denote the set of positive roots by $\Delta^{+}$. Let $\Delta_{\Pi_{1}}$ be the set of nonzero roots of $K$ relative to $T$ and $\Pi_{1}=\left\{\alpha_{i_{1}}, \alpha_{i_{2}}, \cdots, \alpha_{i_{r}}\right\}$ the subset of $\Pi$ which generates $\Delta_{I_{1}}$. If we denote the set of positive roots of $\Delta_{\Pi_{1}}$ by $\Delta_{\Pi_{1}}$, then we have $\Delta_{\Pi_{1}}^{+}=\Delta^{+} \cap \Delta_{\Pi_{1}}$.

Let $G^{c}$ and $K^{c}$ be complexifications of $G$ and $K$, respectively. Let $L$ be the parabolic subgroup of $G^{C}$ such that its Lie algebra is generated by the Lie algebra of $K^{c}$ and $\left\{E_{\alpha} ; \alpha \in \Delta^{+} \backslash \Delta_{\Pi_{1}}^{+}\right\}$. Here $E_{\alpha}$ denotes the root vector of $\alpha \in \Delta$. Then we see

$$
G / K \cong G^{c} / L
$$

as $C^{\infty}$-manifolds. So $G / K$ is a homogeneous complex manifold by this holomorphic structure. Moreover it has a $G$-invariant Kähler metric ([2]). Thus we get a Kähler $C$-space $G / K$. Conversely every Kähler $C$-space can be described as above. Also we can construct the Kähler $C$-space from a pair $\left(\Pi, \Pi_{1}\right)$, where $\Pi$ is a fundamental system of roots and $\Pi_{1}$ is a subset of $\Pi$ ([10] and [2]). 
Let $(\rho, V)$ be an irreducible finite dimensional complex representation of $K$ with highest weight $\hat{\rho}$. We denote by $\left\{\widetilde{\varpi}_{1}, \varpi_{2}, \cdots, \varpi_{l}\right\}$ the system of fundamental weights of $\Pi$. Then the highest weight $\hat{\rho}$ of $(\rho, V)$ can be written as follows :

$$
\hat{\rho}=n_{1} \widetilde{\sigma}_{1}+n_{2} \varpi_{2}+\cdots+n_{l} \widetilde{\sigma}_{l},
$$

where $n_{1}, n_{2}, \cdots, n_{l}$ are integers and if $\alpha_{i} \in \Pi_{1}$ then $n_{i} \geqq 0$.

In this case we can uniquely extend $(\rho, V)$ to a holomorphic representation $\left(\rho_{L}, V\right)$ of $L([3])$. We put

Then

$$
\begin{aligned}
& E_{\rho}=G \times{ }_{\rho} V, \\
& E_{\rho_{L}}=G^{c} \times{ }_{\rho_{L}} V .
\end{aligned}
$$

$$
E_{\rho} \cong E_{\rho_{L}}
$$

as $C^{\infty}$-vector bundles. We regard $E_{\rho}$ as a holomorphic vector bundle by the isomorphism above unless otherwise stated. Also if $(\rho, V)$ is irreducible, there is a unique $G$-invariant Hermitian structure $h$ up to a homothety and $\left(E_{\rho}, h\right)$ is an irreducible Einstein-Hermitian vector bundle ([8]). Therefore we consider $E_{\rho}$ as an irreducible Einstein-Hermitian vector bundle if $(\rho, V)$ is irreducible. For more details about an Einstein-Hermitian vector bundle, we refer the reader to [7].

By $\operatorname{End}\left(E_{\rho}\right)$ we denote the endomorphism bundle of $E_{\rho}$. Let $\operatorname{sil}\left(E_{\rho}\right)$ be the subbundle of $E_{\rho}$ which consists of trace free endomorphisms. By definition of $\operatorname{End}\left(E_{\rho}\right)$ and $\mathfrak{g l}\left(E_{\rho}\right)$,

$$
\begin{aligned}
& \operatorname{End}\left(E_{\rho}\right) \cong G \times{ }_{\rho \otimes \rho *} \operatorname{End}(V), \\
& \mathfrak{g l}\left(E_{\rho}\right) \cong G \times{ }_{\rho \otimes \rho *} \mathfrak{g l}(V),
\end{aligned}
$$

where $\operatorname{End}(V)$ is the linear space of endomorphisms and $\mathfrak{g l}(V)$ is the subspace of $\operatorname{End}(V)$ consisting of trace free endomorphisms. Thus

$$
\operatorname{End}(V)=V \otimes V^{*},
$$

where $V^{*}$ is the dual space of $V$. And $K$ acts $\operatorname{End}(V)$ by the tensor product representation $\left(\rho \otimes \rho^{*}, V \otimes V^{*}\right)$ where $\left(\rho^{*}, V^{*}\right)$ is the dual representation of $(\rho, V)$. By the way $\mathfrak{g l}(E)$ is invariant by $K$. Thus $K$ acts $\mathfrak{g l}(V)$ via the action for $\operatorname{End}(V)$.

Finally we denote the Dolbeault cohomology groups of $\operatorname{End}\left(E_{\rho}\right)$ and $\operatorname{gl}\left(E_{\rho}\right)$ by $H^{p, q}(G / K, \operatorname{End}(\rho))$ and $H^{p, q}\left(G / K, \mathfrak{g l}\left(E_{\rho}\right)\right)$, respectively. We set

$$
\begin{aligned}
& h^{p, q}\left(\operatorname{End}\left(E_{\rho}\right)\right)=\operatorname{dim} H^{p, q}\left(G / K, \operatorname{End}\left(E_{\rho}\right)\right), \\
& h^{p, q}\left(\operatorname{gl}\left(E_{\rho}\right)\right)=\operatorname{dim} H^{p, q}\left(G / K, \operatorname{sl}\left(E_{\rho}\right)\right) .
\end{aligned}
$$




\section{Main Results}

We continue with the notation and the situation in $\S 2$. Let $G / K$ be a Kähler $C$-space where $G$ is a compact simply connected semisimple Lie group and $K$ is the centralizer of a toral subgroup of $G$.

LEMMA 2. Let $(\rho, V)$ be an irreducible complex representation of $K$. Then the restriction of $\left(\rho \otimes \rho^{*}, V \otimes V^{*}\right)$ to the center of $K$ is trivial.

Proof. Let $Z_{K}$ be the center of $K$ and $K^{\prime}$ the semisimple part of $K$. Then

$$
\varphi: Z_{K} \times K^{\prime} \rightarrow K, \quad(z, k) \mapsto z k
$$

is a Lie group homomorphism with kernel $Z_{K} \cap K^{\prime}$. Then $(\rho \circ \varphi, V)$ is a representation of the direct product Lie group $Z_{K} \times K^{\prime}$ on $V$. We note that

$$
\left.\rho \circ \varphi\right|_{z_{K}}=\left.\rho\right|_{z_{K}},\left.\quad \rho \circ \varphi\right|_{K^{\prime}}=\left.\rho\right|_{K^{\prime}} .
$$

Because of irreducibility of $(\rho, V),(\rho \circ \varphi, V)$ is also irreducible. So there are irreducible representations $\left(\rho_{Z_{K}}, V_{Z_{K}}\right)$ of $Z_{K}$ and $\left(\rho_{K^{\prime}}, V_{K^{\prime}}\right)$ of $K^{\prime}$ such that

$$
\left(\rho_{Z_{K}} \otimes \rho_{K^{\prime}}, V_{Z_{K}} \otimes V_{K^{\prime}}\right) \cong(\rho \circ \varphi, V),
$$

where $\left(\rho_{Z_{K}} \otimes \rho_{K^{\prime}}, V_{Z_{K}} \otimes V_{K^{\prime}}\right)$ denotes the exterior tensor product representation of $\left(\rho_{Z_{K}}, V_{Z_{K}}\right)$ and $\left(\rho_{K^{\prime}}, V_{K^{\prime}}\right)$. By irreducibility of $\left(\rho_{Z_{K}}, V_{Z_{K}}\right), V_{Z_{K}}$ is a one dimensional space. Then the tensor product $\left(\rho_{Z_{K}} \otimes \rho_{Z_{K}}^{*}, V_{Z_{K}} \otimes V_{Z_{K}}^{*}\right)$ is isomorphic to the trivial representation.

COROLlARY 3. Let $G$ be a compact semisimple lie group and $K=T$ be a maximal torus of $G$. Let $(\rho, V)$ be an irreducible complex representation of $T$. Then

In particular,

$$
\begin{aligned}
& \operatorname{End}\left(E_{\rho}\right) \cong G / T \times \boldsymbol{C}, \\
& \mathfrak{g l}\left(E_{\rho}\right) \cong G / T \times\{0\} .
\end{aligned}
$$

$$
\begin{aligned}
& h^{0, p}\left(\operatorname{End}\left(E_{\rho}\right)\right)= \begin{cases}1, & \text { for } p=0 \\
0, & \text { for } p \geqq 1,\end{cases} \\
& h^{0, p}\left(\mathfrak{g l}\left(E_{\rho}\right)\right)=0, \text { for } p \geqq 0 .
\end{aligned}
$$

PROOF. We note that any irreducible complex representation space of a torus is one dimensional. From Lemma 1 it is easy to see that $\operatorname{End}\left(E_{\rho}\right)$ is trivial. And also it is easy to see that Hodge numbers of $\operatorname{End}\left(E_{\rho}\right)$ and $\operatorname{al}\left(E_{\rho}\right)$ are as stated above (for example, by means of Bott's generalized Borel-Weil 
theorem ([3, Theorem IV']).

Q.E.D.

Next we consider the case of $\operatorname{rank} G=2$. In this case the fundamental system of roots $\Pi$ is $\left\{\alpha_{1}, \alpha_{2}\right\}$. And if $G / K$ is a Kähler $C$-space then the corresponding $\Pi_{1}$ as in section 2 is $\left\{\alpha_{1}, \alpha_{2}\right\},\left\{\alpha_{1}\right\},\left\{\alpha_{2}\right\}$ or $\phi$. Furthermore a compact simple Lie group $G$ is of type $A_{2}, B_{2}$, or $G_{2}$ in this case. If $G$ is of classical type then corresponding Kähler $C$-spaces are

$$
\begin{array}{ll}
G / K \cong S U(3) / S(U(2) \times U(1)) \cong P_{2} C & \text { if } G=A_{2} \text { and } \Pi_{1}=\left\{\alpha_{1}\right\}, \\
G / K \cong S U(3) / S(U(1) \times U(2)) \cong P_{2} C & \text { if } G=A_{2} \text { and } \Pi_{1}=\left\{\alpha_{2}\right\}, \\
G / K \cong S p(2) /(U(1) \times S p(1)) \cong P_{3} C & \text { if } G=B_{2} \text { and } \Pi_{1}=\left\{\alpha_{1}\right\}, \\
G / K \cong S O(5) /(U(1) \times S O(3)) \cong Q_{3}(C) & \text { if } G=B_{2} \text { and } \Pi_{1}=\left\{\alpha_{2}\right\} .
\end{array}
$$

It is clear that the first case is isomorphic to the second one in the above. And we note that if $\Pi_{1}=\left\{\alpha_{1}, \alpha_{2}\right\}$ then $K=T$ is a maximal torus of $G$, i.e., it is the case of Corollary 3. Also if $\Pi=\phi$ then $K=G$, i. e., $G / K$ consists of a one point $\{0\}$.

Then we state the main theorem.

THEOREM 4. Let $G$ be a compact simply connected simple Lie group with rank $G=2$. Let $\Pi=\left\{\alpha_{1}, \alpha_{2}\right\}$ be a fundamental system of roots relative to $a$ maximal torus $T$ of $G$. We put $\Pi_{1}=\left\{\alpha_{i}\right\}(i=1,2)$. We denote by $K$ the analytic subgroup of $G$ with maximal rank which corresponds to $\Pi_{1}$. Let $(\rho, V)$ be an irreducible complex representation of $K$ with highest weight $\hat{\rho}=n_{1} \varpi_{1}+n_{2} \varpi_{2}$, where $\left\{\varpi_{1}, \varpi_{2}\right\}$ is the system of fundamental weights. Then the Hodge number $h^{0 . p}\left(\operatorname{End}\left(E_{\rho}\right)\right)$ is as follows:

(I) If $G$ is of type $A_{2}$ and $\Pi_{1}=\left\{\alpha_{2}\right\}$ then

$$
h^{0 . p}\left(\operatorname{End}\left(E_{\rho}\right)\right)= \begin{cases}1, & \text { if } p=0, \\ \frac{1}{2} \sum_{k=1}^{n_{2}}(2 k+1)(k+2)(k-1), & \text { if } p=1, \\ 0, & \text { if } p \geqq 2 .\end{cases}
$$

(II) If $G$ is of type $B_{2}$ and $\Pi_{1}=\left\{\alpha_{1}\right\}$ then

$$
h^{0 . p}\left(\operatorname{End}\left(E_{\rho}\right)\right)= \begin{cases}1, & \text { if } p=0, \\ \frac{1}{3} \sum_{k=1}^{n_{1}}(2 k+3)(2 k+1)(2 k-1), & \text { if } p=1, \\ 0, & \text { if } p \geqq 2 .\end{cases}
$$


(III) If $G$ is of type $B_{2}$ and $\Pi_{1}=\left\{\alpha_{2}\right\}$ then

$$
h^{0 . p}\left(\operatorname{End}\left(E_{\rho}\right)\right)= \begin{cases}1, & \text { if } p=0, \\ \frac{1}{2} \sum_{k=1}^{n_{2}}(2 k-1)(k+2)(k-1), & \text { if } p=1, \\ 0, & \text { if } p \geqq 2 .\end{cases}
$$

(IV) If $G$ is of type $G_{2}$ and $\Pi_{1}=\left\{\alpha_{1}\right\}$ then

$$
h^{0, p}\left(\operatorname{End}\left(E_{\rho}\right)\right)= \begin{cases}1, & \text { if } p=0, \\ 7, & \text { if } p=1 \text { and } n_{1}=2, \\ 21, & \text { if } p=1 \text { and } n_{1} \geqq 3, \\ \frac{1}{40} \sum_{k=5}^{n_{1}}(2 k+1)(k+5)(k+2)(k-1)(k-4), & \text { if } p=2, \\ 0, & \text { otherwise. }\end{cases}
$$

(V) If $G$ is of type $G_{2}$ and $\Pi_{1}=\left\{\alpha_{2}\right\}$ then

$$
h^{0, p}\left(\operatorname{End}\left(E_{\rho}\right)\right)= \begin{cases}1, & \text { if } p=0, \\ 2261, & \text { if } p=1, \\ \frac{1}{24} \sum_{k=2}^{n_{2}}(21 k-2)(18 k-1)(13 k-1)(8 k-1)(3 k-1) k, \\ 0, & \text { if } p=2,\end{cases}
$$

THEOREM 5. Under the same assumption of Theorem 4, the Hodge number $h^{0, p}\left(\mathfrak{g l}\left(E_{\rho}\right)\right)$ is equal to

$$
h^{0, p}\left(\mathfrak{g l}\left(E_{\rho}\right)\right)=\left\{\begin{array}{cl}
0, & \text { if } p=0, \\
h^{0, p}\left(\operatorname{End}\left(E_{\rho}\right)\right), & \text { if } p \geqq 1,
\end{array}\right.
$$

for every cases ( I ) (V) in Theorem 4.

We shall prove these theorems in the next section. We state some consequences of these results here. If $h^{0,0}\left(\mathfrak{g l}\left(E_{\rho}\right)\right)=h^{0.2}\left(\mathfrak{g l}\left(E_{\rho}\right)\right)=0$, then we can identify $H^{0,1}\left(G / K\right.$, $\left.\operatorname{End}\left(E_{\rho}\right)\right)$ with the tangent space at the homogeneous structures of the moduli space of $E_{\rho}([6],[9]$ and $[7$, Chapter VII $])$. Then we get the following corollary.

COROLLARY 6. Under the same assumption of theorems above, the dimension of the moduli space of irreducible Einstein-Hermitian structures is as follows:

( I ) If $G$ is of type $A_{2}$ and $\Pi_{1}=\left\{\alpha_{2}\right\}$ then

$$
\frac{1}{2} \sum_{k=1}^{n_{2}}(2 k+1)(k+2)(k-1) \text {. }
$$


(II) If $G$ is of type $B_{2}$ and $\Pi_{1}=\left\{\alpha_{1}\right\}$ then

$$
\frac{1}{3} \sum_{k=1}^{n_{1}}(2 k+3)(2 k+1)(2 k-1) \text {. }
$$

(III) If $G$ is of type $B_{2}$ and $\Pi_{1}=\left\{\alpha_{2}\right\}$ then

$$
\frac{1}{2} \sum_{k=1}^{n_{2}}(2 k-1)(k+2)(k-1) \text {. }
$$

(IV) If $G$ is of type $G_{2}$ and $\Pi_{1}=\left\{\alpha_{1}\right\}$ then

$$
\left\{\begin{aligned}
0, & \text { for } n_{1}=0 \text { or } 1 \\
7, & \text { for } n_{1}=2 \\
21, & \text { for } n_{1}=3 \text { or } 4
\end{aligned}\right.
$$

(V) If $G$ is of type $G_{2}$ and $\Pi_{1}=\left\{\alpha_{2}\right\}$ then

$$
\left\{\begin{aligned}
0, & \text { for } n_{2}=0, \\
261, & \text { for } n_{2}=1 .
\end{aligned}\right.
$$

Because of Theorem 5, we see that if $G$ is of classical type, then $h^{0.0}\left(\mathfrak{g l}\left(E_{\rho}\right)\right)=h^{0.2}\left(\mathfrak{g l}\left(E_{\rho}\right)\right)=0$. Moreover we note that if $I I_{1}=\left\{\alpha_{i}\right\}$ then $\operatorname{rank} E_{\rho}$ $=n_{i}+1$ in theorems and corollary above. Also under the same condition, we note that the dimension of the moduli space of $E_{\rho}$ depends only on $n_{i}$. Then we get Theorem 1 from Corollaries 3 and 6 .

\section{Proof of Theorems}

In this sections, we prove Theorems 4 and 5 . The two theorems 4 and 5 are proved at the same time. First we note equations (1) and (2) in $\S 2$. So $\operatorname{End}\left(E_{\rho}\right)$ and $\mathfrak{i l}\left(E_{\rho}\right)$ are defined by representations $\left(\rho \otimes \rho^{*}, V \otimes V^{*}\right)$ and $\left(\rho \otimes \rho^{*}, \mathfrak{g l}(V)\right)$, respectively. Because of Lemma $2, \rho \otimes \rho^{*}$ is trivial on the center of $K$. The semisimple part of $K$ is of type $A_{1}$ in these cases. Thus we can apply the Clebsch-Gordan theorem to the representation $\rho \otimes \rho^{*}$. Therefore we see that if $\Pi_{1}=\left\{\alpha_{i}\right\}$ and the highest weight of $\rho$ is given by $\hat{\rho}=n_{1} \varpi+n_{2} \varpi$, then the highest weight which corresponds to each irreducible component of $\left(\rho \otimes \rho^{*}, \operatorname{End}(V)\right)$ are given by

$$
n_{i} \alpha_{i},\left(n_{i}-1\right) \alpha_{i},\left(n_{i}-2\right) \alpha_{i}, \cdots, \alpha_{i}, 0 .
$$

Also under the same assumption the highest weight which corresponds to each irreducible component of $\left(\rho \otimes \rho^{*}, \mathfrak{g}(V)\right)$ are given by

$$
n_{i} \alpha_{i},\left(n_{i}-1\right) \alpha_{i},\left(n_{i}-2\right) \alpha_{i}, \cdots, 2 \alpha_{i}, \alpha_{i} .
$$


Let $\left(\rho_{k}, V_{k}\right)$ be the complex irreducible representation of $K$ with highest weight $\hat{\rho}_{k}=k \alpha_{i}$ and we put $E_{\rho_{k}}=G \times \rho_{\rho_{k}} V_{k}$. Then (4) and (5) imply the following, respectively :

$$
\begin{aligned}
& H^{0, p}\left(G / K, \operatorname{End}\left(E_{\rho}\right)\right)=\bigoplus_{k=0}^{n_{2}} H^{0, p}\left(G / K, E_{\rho_{k}}\right), \\
& H^{0, p}\left(G / K, \mathfrak{g l}\left(E_{\rho}\right)\right)=\bigoplus_{k=1}^{n_{2}} H^{0, p}\left(G / K, E_{\rho_{k}}\right) .
\end{aligned}
$$

Next we compute the cohomology one by one. We denote by $\delta$ the half of the sum of the positive roots, i.e.,

$$
\delta=\varpi_{1}+\varpi_{2}
$$

in these cases. And we denote by $S_{\alpha}$ the reflection with respect to $\alpha \in \Delta$. We use the tables of root systems in [4] for the following.

Case (I) In this case $G$ is of type $A_{2}$ and $\Pi_{1}=\left\{\alpha_{2}\right\}$. Then we see

$$
\begin{aligned}
\delta+\hat{\rho}_{k} & =\delta+k \alpha_{2} \\
& =-(k-1) \varpi_{1}+(2 k+1) \varpi_{2} .
\end{aligned}
$$

From this, we see that

$$
\delta+\hat{\rho} \text { is } \begin{cases}\text { regular } & \text { if } k \neq 1, \\ \text { singular } & \text { if } k=1\end{cases}
$$

in the sense of [3]. Also we see that

$$
\text { the index of } \delta+\hat{\rho}_{k}= \begin{cases}0, & \text { if } k=0, \\ 1, & \text { if } k \neq 0,1\end{cases}
$$

By the way, we have

$$
S_{\alpha_{1}}\left(\delta+\hat{\rho}_{k}\right)=(k-1) \varpi_{1}+(k+2) \varpi_{2} .
$$

This implies that $S_{\alpha_{1}}\left(\delta+\hat{\rho}_{k}\right)$ is contained in the fundamental Weyl chamber. We put

$$
\lambda_{k}=(k-2) \widetilde{\varpi}_{1}+(k+1) \widetilde{\varpi}_{2}
$$

and $V_{\lambda_{k}}$ denotes the complex irreducible representation space of $G$ with highest weight $\lambda_{k}$. By means of Bott's generalized Borel-Weil theorem ([3, Theorem $\left.\mathrm{IV}^{\prime}\right]$ ), we get

$$
H^{0, p}\left(G / K, E_{\rho_{k}}\right) \cong \begin{cases}V_{\lambda_{k}}, & \text { for } k \geqq 1 \text { and } p=1, \\ \{0\}, & \text { for } k \geqq 1 \text { and } p \neq 1,\end{cases}
$$

and 


$$
H^{0 . p}\left(G / K, E_{\rho_{k}}\right) \cong \begin{cases}C, & \text { for } k=0 \text { and } p=0, \\ \{0\}, & \text { for } k=0 \text { and } p \geqq 1 .\end{cases}
$$

as complex $G$-spaces. We have

$$
H^{0, p}\left(G / K, \operatorname{End}\left(E_{\rho}\right)\right) \cong\left\{\begin{array}{cc}
C, & \text { for } p=0, \\
\bigoplus_{k=1}^{n_{2}} V_{\lambda_{k}}, & \text { for } p=1, \\
\{0\}, & \text { for } p \geqq 2,
\end{array}\right.
$$

from equations (6), (8) and (9) and

$$
H^{0, p}\left(G / K, \operatorname{gl}\left(E_{\rho}\right)\right) \cong\left\{\begin{array}{cc}
\bigoplus_{k=1}^{n_{2}} V_{\lambda_{k}}, & \text { for } p=1, \\
\{0\}, & \text { for } p \neq 1,
\end{array}\right.
$$

from equations (7), (8) and (9) as complex $G$-spaces. We can compute $h^{0, p}\left(\operatorname{End}\left(E_{\rho}\right)\right)$ and $h^{0, p}\left(\operatorname{gr}\left(E_{\rho}\right)\right)$ by Weyl's dimension formula for a complex irreducible representation space. Then we obtain theorems in the case (I).

Case (II) In this case $G$ is of type $B_{2}$ and $\Pi_{1}=\left\{\alpha_{1}\right\}$. Then we see

$$
\begin{aligned}
\delta+\hat{\rho}_{k} & =\delta+k \alpha_{2} \\
& =(2 k+1) \varpi_{1}-(2 k-1) \varpi_{2} .
\end{aligned}
$$

From this, we see $\delta+\hat{\rho}_{k}$ is regular for any $k$. Also we see

$$
\text { the index of } \delta+\hat{\rho}_{k}= \begin{cases}0, & \text { for } k=0, \\ 1, & \text { for } k \neq 0 .\end{cases}
$$

By the way, we have

$$
S_{\alpha_{2}}\left(\delta+\hat{\rho}_{k}\right)=2 \varpi_{1}+(2 k-1) \varpi_{2} .
$$

This implies that $S_{\alpha_{2}}\left(\delta+\hat{\rho}_{K}\right)$ is contained in the fundamental Weyl chamber. In the same way as in the case (I), we get

$$
\begin{gathered}
H^{0, p}\left(G / K, \operatorname{End}\left(E_{\rho}\right)\right) \cong\left\{\begin{array}{cc}
C, & \text { for } p=0, \\
\bigoplus_{k=1}^{n_{1}} V_{\lambda_{k}}, & \text { for } p=1, \\
\{0\}, & \text { for } p \geqq 2,
\end{array}\right. \\
H^{0, p}\left(G / K, \mathfrak{g l}\left(E_{\rho}\right)\right) \cong\left\{\begin{array}{cc}
\bigoplus_{k=1}^{n_{1}} V_{\lambda_{k}}, & \text { for } p=1, \\
\{0\}, & \text { for } p \neq 1
\end{array}\right.
\end{gathered}
$$

as complex $G$-spaces. Here

$$
\lambda_{k}=(2 k-1) \varpi_{1}+(2 k-2) \varpi_{2}
$$

and $V_{\lambda_{k}}$ is the irreducible complex representation space of $G$ which corresponds 
to $\lambda_{k}$. We get $h^{0, p}\left(\operatorname{End}\left(E_{\rho}\right)\right)$ and $h^{0, p}\left(\mathfrak{g l}_{\mathcal{L}}\left(E_{\rho}\right)\right)$ by Weyl's dimension formula as before.

Case (III) In this case $G$ is of type $B_{2}$ and $I_{1}=\left\{\alpha_{2}\right\}$. Then we see

$$
\begin{aligned}
\delta+\hat{\rho}_{k} & =\delta+k \alpha_{2} \\
& =-(k-1) \varpi_{1}+(2 k+1) \varpi_{2} .
\end{aligned}
$$

From this, we see

$$
\delta+\hat{\rho}_{k} \text { is } \begin{cases}\text { regular, } & \text { if } k \neq 1, \\ \text { singular, } & \text { if } k=1,\end{cases}
$$

and

$$
\text { the index of } \delta+\hat{\rho}_{k}= \begin{cases}0, & \text { for } k=0, \\ 1, & \text { for } k \neq 0,1 .\end{cases}
$$

By the way, we have

$$
S_{\alpha_{1}}\left(\delta+\hat{\rho}_{k}\right)=(k-1) \varpi_{1}+3 \varpi_{2} .
$$

This implies that $S_{\alpha_{1}}\left(\delta+\hat{\rho}_{k}\right)$ is in the fundamental Weyl chamber. As before, we get

$$
\begin{gathered}
H^{0, p}\left(G / K, \operatorname{End}\left(E_{\rho}\right)\right) \cong\left\{\begin{array}{cc}
C, & \text { for } p=0, \\
\bigoplus_{k=1}^{n_{2}} V_{\lambda_{k}}, & \text { for } p=1, \\
\{0\}, & \text { for } p \geqq 2,
\end{array}\right. \\
H^{0, p}\left(G / K, \operatorname{gl}\left(E_{\rho}\right)\right) \cong\left\{\begin{array}{cc}
\bigoplus_{k=1}^{n_{2}} V_{\lambda_{k}}, & \text { for } p=1, \\
\{0\}, & \text { for } p \neq 1
\end{array}\right.
\end{gathered}
$$

as complex $G$-spaces. Here

$$
\lambda_{k}=(k-2) \varpi_{1}+2 \varpi_{2}
$$

and $V_{\lambda_{k}}$ is the irreducible complex representation space of $G$ which corresponds to $\lambda_{k}$. We get $h^{0, p}\left(\operatorname{End}\left(E_{\rho}\right)\right)$ and $h^{0, p}\left(\mathfrak{g r}\left(E_{\rho}\right)\right)$ by Weyl's dimension formula as before.

Case (IV) In this case $G$ is of type $G_{2}$ and $I I_{1}=\left\{\alpha_{1}\right\}$. Then we see

$$
\begin{aligned}
\delta+\hat{\rho}_{k} & =\delta+k \alpha_{2} \\
& =(2 k+1) \varpi_{1}-(k-1) \varpi_{2} .
\end{aligned}
$$

From this, we see that

$$
\delta+\hat{\rho}_{k} \text { is } \begin{cases}\text { regular, } & \text { if } k \neq 1 \text { and } 4, \\ \text { singular, } & \text { if } k=1 \text { or } 4,\end{cases}
$$

and 


$$
\text { the index of } \delta+\hat{\rho}_{k}= \begin{cases}0, & \text { for } k=0, \\ 1, & \text { for } k=1,2,3 \text { or } 4, \\ 2, & \text { for } k \geqq 5 .\end{cases}
$$

By the way we have,

$$
S_{\alpha_{2}}\left(\delta+\hat{\rho}_{k}\right)=-(k-4) \varpi_{1}+(k-1) \varpi_{2}
$$

and

$$
S_{\alpha_{1}} \circ S_{\alpha_{2}}\left(\delta+\hat{\rho}_{k}\right)=(k-4) \varpi_{1}+3 \varpi_{2} .
$$

This implies that $S_{\alpha_{2}}\left(\delta+\hat{\rho}_{k}\right)$ is contained in the fundamental Weyl chamber if $k=2,3$ and $S_{\alpha_{1}}{ }^{\circ} S_{\alpha_{2}}\left(\delta+\hat{\rho}_{k}\right)$ is contained in the fundamental Weyl chamber if $k \geqq 5$. As before, we get

$$
H^{0 . p}\left(G / K, \operatorname{End}\left(E_{\rho}\right)\right) \cong\left\{\begin{array}{cl}
C, & \text { if } p=0, \\
\{0\}, & \text { if } p=1 \text { and } n_{1}=0,1, \\
V \lambda_{2}, & \text { if } p=1 \text { and } n_{1}=2, \\
V_{\lambda_{2}} \oplus V_{\lambda_{3}}, & \text { if } p=1 \text { and } n_{1} \geqq 3, \\
\bigoplus_{k=5}^{n_{1}} V_{\lambda_{k}}, & \text { if } p=2, \\
\{0\}, & \text { if } p \geqq 3, \\
\{0\}, & \text { if } p=0, \\
\{0\}, & \text { if } p=1 \text { and } n_{1}=0,1, \\
V \lambda_{\lambda_{2}}, & \text { if } p=1 \text { and } n_{1}=2, \\
V_{\lambda_{2}} \oplus V_{\lambda_{3}}, & \text { if } p=1 \text { and } n_{1} \geqq 3, \\
\bigoplus_{1} V_{\lambda_{k}}, & \text { if } p=2, \\
\{0\}, & \text { if } p \geqq 3
\end{array}\right.
$$

as complex $G$-spaces. Here

$$
\lambda_{k}= \begin{cases}-(k-3) \varpi_{1}+(k-2) \varpi_{2}, & \text { for } k=2,3, \\ (k-5) \varpi_{1}+2 \varpi_{2}, & \text { for } k \geqq 5,\end{cases}
$$

And $V_{\lambda_{k}}$ is the irreducible complex representation space of $G$ which corresponds to $\lambda_{k}$. We can compute $h^{0 \cdot p}\left(\operatorname{End}\left(E_{\rho}\right)\right)$ and $h^{0 \cdot p}\left(\mathscr{I l}\left(E_{\rho}\right)\right)$ by Weyl's dimension formula as before.

Case (V) In this case $G$ is of type $G_{2}$ and $\Pi_{1}=\left\{\alpha_{2}\right\}$. Then we see

$$
\begin{aligned}
\delta+\hat{\rho}_{k} & =\delta+k \alpha_{2} \\
& =-(3 k-1) \varpi_{1}+(2 k+1) \varpi_{2} .
\end{aligned}
$$

From this, we see that 
and

$$
\delta+\hat{\rho}_{k} \text { is } \begin{cases}\text { regular, } & \text { if } k \neq 2, \\ \text { singular, } & \text { if } k=2 .\end{cases}
$$

$$
\text { the index of } \delta+\hat{\rho}_{k}= \begin{cases}0, & \text { for } k=0, \\ 1, & \text { for } k=1, \\ 2, & \text { for } k \geqq 2 .\end{cases}
$$

By the way, we have

$$
S_{\alpha_{1}}\left(\delta+\hat{\rho}_{k}\right)=(3 k-1) \varpi_{1}+5 k \varpi_{2} .
$$

This implies that $S_{\alpha_{1}}\left(\delta+\hat{\rho}_{k}\right)$ is contained in the fundamental Weyl chamber. As before, we get

$$
\begin{aligned}
H^{0, p}\left(G / K, \text { End }\left(E_{\rho}\right)\right) \cong\left\{\begin{array}{cl}
C, & \text { for } p=0, \\
\{0\}, & \text { for } p=1 \text { and } n_{2}=0, \\
V_{\lambda_{1}}, & \text { for } p=1 \text { and } n_{2} \geqq 1, \\
\bigoplus_{k=2}^{n_{2}} V_{\lambda_{k}}, & \text { for } p=2, \\
\{0\}, & \text { for } p \geqq 3,
\end{array}\right. \\
H^{0, p}\left(G / K, \operatorname{gr}\left(E_{\rho}\right)\right) \cong\left\{\begin{array}{cl}
\{0\}, & \text { for } p=0, \\
\{0\}, & \text { for } p=1 \text { and } n_{2}=0, \\
V_{\lambda_{1}}, & \text { for } p=1 \text { and } n_{2} \geqq 1, \\
\bigoplus_{k=2} V_{\lambda_{k}}, & \text { for } p=2, \\
\{0\}, & \text { for } p \geqq 3
\end{array}\right.
\end{aligned}
$$

as complex $G$-spaces. Here

$$
\lambda_{k}=(3 k-2) \varpi_{1}+(5 k-1) \varpi_{2},
$$

and $V_{\lambda_{k}}$ is the irreducible complex representation space of $G$ which corresponds to $\lambda_{k}$. We can compute $h^{0, p}\left(\operatorname{End}\left(E_{\rho}\right)\right)$ and $h^{0, p}\left(\mathfrak{g l}\left(E_{\rho}\right)\right)$ by Weyl's dimension formula as before.

Now we complete the proof of Theorems 4 and 5 .

REMARK. We only write the dimensions in the statement of Theorems and Corollary, but we get the cohomology groups and the tangent spaces of moduli spaces as the representation space of $G$. Indeed, we have determined the highest weight of each irreducible component in the proof. 


\section{References}

[1] J.F. Adams, Lectures on Lie Groups, Benjamin, New York, 1969.

[2] A. Borel, Kählerian coset spaces of semi-simple Lie groups, Proc. Nat. Acad. Sci. U.S. A. 40 (1954), 1147-1151.

[3] R. Bott, Homogeneous vector bundles, Ann. of Math. 66 (1957), 203-248.

[4] N. Bourbaki, Group et Algèbre de Lie, Chapitre 4, 5 et 6, Hermann, Paris, 1968.

[5] T. Bröker and T. Dieck, Representations of Compact Lie Groups, Springer-Verlag, New York, 1985.

[6] H. J. Kim, Moduli of Hermite-Einstein vector bundles, Math. Z. 195 (1987), 143150.

[7] S. Kobayashi, Differential Geometry of Complex Vector Bundles, Iwanami Shoten, Tokyo, 1987.

[8] S. Kobayashi, Homogeneous vector bundles and stability, Nagoya Math J. 101 (1986), 37-54.

[9] Lübke and Okonek, Moduli spaces of simple bundles and Einstein-Hermitian connections, Math. Ann. 276 (1987), 663-674.

[10] H.C. Wang, Closed manifolds with homogeneous complex structure, Amer. J. Math. 76 (1954), 1-32.

Institute of Mathematics

University of Tsukuba

Tsukuba, Ibaraki, 305

Japan 